Urbi Garay*

Manuel Hernández"*

Carlos Rivillo**

Instituto de Estudios Superiores de Administración (IESA) Caracas, Venezuela

Recibido: 26 de noviembre de 2015 Concepto de evaluación: 30 de junio de 2017 Aprobado: 6 de julio de 2017

Artículo de investigación (C) 2017 Universidad Católica de Colombia. Facultad de Ciencias

Económicas y Administrativas. Todos los derechos reservados

\footnotetext{
* Ph.D. en Finanzas, M. A. en Economía Internacional y Desarrollo, Economista. Profesor titular de Finanzas del Instituto de Estudios

Superiores de Administración (IESA), Caracas, Venezuela. Dirección de correspondencia: IESA POBA International \# 646, P.O. Box 02-5255, Miami, FI, 33102. Correo electrónico: urbi.garay@iesa.edu.ve

** Magíster en Finanzas e Ingeniero Industrial. Egresado del Instituto de Estudios Superiores de Administración (IESA), Caracas, Venezuela. Correo electrónico: manuel.hernandez@iesa.edu.ve
}

Finanz. polit. econ., ISSN: 2248-6046, Vol. 9, No. 2, julio-diciembre, 2017, pp. 373-396

http://dx.doi.org/10.14718/revfinanzpolitecon.2017.9.2.8

\section{Variables microeconómicas de los fondos de fondos de cobertura (FFC) y su desempeño durante la crisis financiera global 2008-2009}

\section{RESUMEN}

Este trabajo examina el comportamiento de las variables microeconómicas de los fondos de fondos de cobertura (FFC) durante un periodo previo a la crisis financiera global 2008-2009, y analiza si dichas variables explican el desempeño de los FFC durante tal crisis. La magnitud y la severidad de la crisis representan un evento ideal para determinar las características de los FFC que lograron sobrevivirla. Mediante la aplicación de un modelo de regresión Probit, se concluyó que las siguientes variables explican la probabilidad de sobrevivencia de los FFC: rendimiento promedio previo, desviación estándar de los rendimientos, comisión fija, comisión por incentivo y curtosis de los rendimientos.

Palabras clave: fondos de fondos de cobertura, crisis financiera 20082009, Probit.

JEL: G12, G15, G24.

Microeconomic variables of funds of hedge funds

(FHF) and their performance during the global financial crisis of 2008-2009

\section{ABSTRACT}

This paper examines the behavior of the microeconomic variables of funds of hedge funds (FHF) during a period prior to the global financial crisis of 2008-2009, and analyzes whether these variables explain the performance of FHF during such crisis. Given its magnitude and severity, this crisis constitutes an ideal event to determine the characteristics of FHF that managed to survive it. Using a Probit regression model, it was concluded that the following variables explain the survival probability of FHF: previous average yield, standard deviation of yields, fixed commission, incentive commission, and yield kurtosis.

Keywords: funds of hedge funds, financial crisis of 2008-2009, Probit. 
*** Magíster en Finanzas y Contador Público. Egresado del Instituto de Estudios Superiores de Administración (IESA), Caracas, Venezuela. Correo electrónico: carlos.rivillo@iesa.edu.ve

\section{Ariáveis microeconômicas dos fundos de fundos multimercados (hedge funds) e seu desempenho durante a crise financeira global 2008-2009}

\section{RESUMO}

Este trabalho examina o comportamento das variáveis microeconômicas dos fundos de fundos multimercados (hedge funds) durante o período prévio à crise financeira global de 2008-2009 e analisa se essas variáveis explicam o desempenho dos fundos durante tal crise. A magnitude e gravidade da crise representam um evento ideal para determinar as características dos fundos hedge funds que conseguiram sobreviver a ela. Mediante a aplicação de um modelo de regressão Probit, concluiu-se que as seguintes variáveis explicam a probabilidade de sobrevivência desses fundos: retorno médio prévio, desviopadrão dos rendimentos, comissão fixa, comissão por incentivo e curtose do retorno de investimentos.

Palavras-chave: crise financeira 2008-2009, fundos de fundos multimercados (hedge funds), Probit 


\section{INTRODUCCIÓN}

Los fondos de cobertura (FC) son sociedades privadas de inversión limitada que ofrecen oportunidades de inversión atractivas, al tomar posiciones en una amplia variedad de instrumentos financieros y mercados globales, típicamente no disponibles para los inversionistas tradicionales (Stulz, 2007). Por otra parte, los fondos de fondos de cobertura (FFC) son vehículos de inversión que captan el capital de varios inversionistas y lo distribuyen entre distintos FC, con el objetivo de diversificarse a través de los distintos estilos, estrategias y gerentes de fondos. Al invertir en un FFC, los inversionistas delegan el manejo de su portafolio al gerente del FFC. Esta gerencia está encargada de seleccionar los FC individuales, desarrollar y completar una diligencia debida sobre ellos, monitorear el riesgo y el retorno, gestionar el portafolio en general y reportar los resultados obtenidos a sus inversionistas.

Se considera que Alfred W. Jones comenzó el primer FC en 1949, al cual condujo hasta los años setenta. Jones recaudó USD 60.000 e invirtió USD 40.000 de su propio capital para seguir una estrategia de invertir en acciones comunes y cubrir las posiciones con ventas en corto de otras acciones de otras empresas. A partir de estos modestos comienzos, el volumen de activos gestionados a través de FC creció sostenidamente, especialmente a finales del siglo pasado. A finales de 1993, los FC gestionaban el equivalente a menos del $4 \%$ de los activos administrados por fondos mutuales de inversión; para el 2014, este porcentaje había aumentado a más del 10\%. En 1990, menos de USD 50.000 millones eran gestionados por los FC; en 2014, estos fondos manejaban más de 2 billones de dólares, recuperándose así de la importante caída sufrida durante la crisis financiera global de 2008-2009.

La industria de los FC no escapó a la crisis financiera que estalló a finales de 2008. Este estudio pretende analizar el comportamiento de algunas variables microeconómicas de los FFC durante un periodo previo a la crisis, con el objeto de analizar si podían predecir la probabilidad de sobrevivencia de este tipo de fondos durante la crisis 2008-2009.
La gravedad de la crisis en mención representa un evento externo idóneo para estudiar las características de los FFC que lograron sobrevivirla, es decir, permanecer activos. La principal innovación del trabajo consiste en el uso del modelo Probit como herramienta para predecir la probabilidad de que los FFC - en lugar de los FC, ya estudiados por algunos autores como Xu, Liu y Loviseck (2011) — hubieran sobrevivido a la crisis. Además, en el modelo Probit propuesto se consideran algunas variables que no habían sido incluidas en el trabajo de los autores referidos.

El resto del presente trabajo está constituido por las siguientes secciones: en la próxima se presentan algunas definiciones y el marco teórico que fundamenta esta investigación; luego se detalla la hipótesis que subyace a este trabajo y se describen las variables que se utilizarán para la comprobación de dicha hipótesis; en la siguiente sección se describen los datos y la metodología utilizada y, a continuación, se presenta el análisis de los resultados obtenidos. En la última sección del trabajo se presentan las conclusiones y se esbozan algunas posibles extensiones del trabajo.

\section{DEFINICIONES Y REVISIÓN DE LA LITERATURA}

\section{Fondos de cobertura}

Los FC se pueden comparar con los fondos mutuales, con los que tienen algunas similitudes $y$ bastantes diferencias. Ambos vehículos de inversión captan dinero de terceros con la finalidad de realizar inversiones, siguiendo lo indicado en el prospecto respectivo; ofrecen así la posibilidad de diversificar el portafolio en la clase de activos en la cual invierta el fondo mutual, o en los activos o la estrategia que siga el FC.

Las principales diferencias entre los fondos mutuales y los FC se señalan a continuación. En primer lugar, los fondos mutuales están dirigidos al público general (cualquiera puede invertir en estos vehículos desde montos muy bajos), mientras que 
los FC están dirigidos a inversionistas "sofisticados", es decir, a inversionistas que cumplan una serie de requisitos bastante estrictos definidos por el ente regulador (principalmente, monto de patrimonio total y activos financieros totales del inversionista).

En segundo lugar, los fondos mutuales están inscritos y regulados por las comisiones o superintendencias de valores de los países en los que están inscritos, mientras que los FC no tienen que registrarse ante las comisiones de valores (excepto aquellos FC que tengan activos por más de \$150 millones, en el caso de los Estados Unidos, los cuales sí tendrían que registrarse en la Securities and Exchange Commission [SEC]).

En tercer lugar, los fondos mutuales tienen severas limitaciones en cuanto a la posibilidad de realizar ventas en corto, apalancarse y hacer uso de productos derivados. Por el contrario, los FC no tienen mayores limitaciones con respecto a este tipo de operaciones, más allá de las que los inversionistas en el fondo hayan aceptado y considerado las leyes de mercado de capitales de los países en los cuales realicen operaciones. Como resultado, los FC cuentan con niveles de flexibilidad mucho mayores que los fondos mutuales.

\section{Fondos de fondos de cobertura}

Como se mencionó, los fondos de fondos de cobertura (FFC) son vehículos que a su vez invierten en FC. De acuerdo con Hedge Fund Research Inc., para el tercer trimestre del 2008 había 2540 FFC reportados en las bases de datos, versus 538 FFC al final del 2000, y apenas $80 \mathrm{FFC}$ al final de 1990'. Para el tercer trimestre del 2008, los FFC contaban con activos de alrededor de unos USD 772.000 millones, más de un tercio del total de activos manejados por la industria de los FC, estimados en unos USD 1,8 billones para ese momento. Después de la crisis, el número de FFC se ha mantenido en alrededor de 2000 fondos (hasta 2013).

$1 \quad$ Las siguientes son algunas de las bases de datos a las que los fondos de cobertura y los FFC deciden reportar sus resultados financieros: CISDM-Morningstar, Hedge Fund Research (HFR), Credit Suisse y Bloomberg.
Un FFC ofrece a los inversionistas la ventaja de realizar la debida diligencia profesional, la selección de gerentes y la supervisión de los FC en los cuales invierte. Los FC suelen tener elevados niveles de inversión mínima, lo que restringe la capacidad de muchos inversores de diversificar sus carteras, dependiendo de la cantidad de dinero asignado para su fondo. Con un FFC, los inversionistas con un capital limitado pueden acceder a varios FC con una sola inversión y logran así una diversificación instantánea. La selección de varios fondos puede proporcionar una mayor estabilidad, es decir, una menor volatilidad de los rendimientos al distribuir los activos en un rango más amplio de distintas estrategias. En lugar de asumir el riesgo de seleccionar un gerente individual, el FFC ofrece una cartera de gerentes con una sola inversión.

Entre las desventajas de los FFC se encuentra que las comisiones de estos vehículos de inversión son relativamente altas, puesto que es necesario erogar, primero, una comisión al FC y, segundo, el FFC habrá pagado una comisión a cada FC en el que haya invertido (ambas comisiones contienen una porción fija o comisión por gerencia del fondo, y una comisión que es un porcentaje del desempeño obtenido o comisión por incentivo). Por otra parte, los FC que gestionen activos totales menores a 150 millones de dólares no están obligados a registrarse ante la Comisión Nacional de Valores de los Estados Unidos y, por lo general, se venden en ofertas privadas. Esto significa que las posiciones dentro de los FC no tienen que ser informadas de forma pública, mientras que las de los fondos mutuales sí deben hacerlo. No obstante, los FC siguen estando sujetos a las responsabilidades básicas de fiduciario como asesores de inversiones registrados.

\section{Riesgos de los fondos de fondos de cobertura}

La inversión en FFC es más complicada e implica un mayor riesgo en algunos aspectos cuando se comparan con las inversiones financieras tradicionales. A continuación, se comentan algunos de estos riesgos. 
En primer lugar, la mayoría de los FC, vehículo en el cual invierten los FFC, cuentan con un periodo durante el cual el inversionista está comprometido a no retirar el dinero (este periodo puede inclusive durar varios años). Los FC suelen limitar las oportunidades para redimir o comprar acciones, por ejemplo, a solo una vez por trimestre, semestre o incluso por año. Esto reduce la capacidad de un inversionista de retirar dinero de un fondo en tiempos de turbulencia y alta volatilidad en los mercados.

Estos límites en el porcentaje de capital que puede ser retirado en una fecha de rescate también reducen la capacidad de los inversionistas de los FC para salir de un fondo. Esta característica es cada vez más común. Los gerentes de FC necesitan de algunas de estas restricciones para poder reducir la variabilidad en la entrada y salida de fondos a la cartera, al igual que para protegerse ante el riesgo de que ocurran retiros masivos de capitales del fondo en medio de una crisis. Esto es más comúnmente empleado cuando los mercados están cayendo, que es exactamente el momento en que un inversionista desea poder liquidar sus inversiones en estos fondos. Evidentemente, todas estas restricciones al retiro de dinero invertido en los FC afectan los FFC.

Un FFC depende de la experticia y de la habilidad del gerente del fondo para seleccionar los FC que se desempeñarán bien. Si este no es el objetivo del FFC, sus retornos se verán afectados de forma negativa. Así, las comisiones sobre el rendimiento del FFC pueden motivar a sus gerentes a asumir mayores riesgos con la esperanza de generar un retorno mayor para ellos y sus inversionistas. Si un gerente obtiene una porción sustancial de las ganancias de capital de un fondo, pudiera entonces verse tentado a tomar riesgos innecesarios con el fin de beneficiarse de las posibilidades de obtener una elevada rentabilidad y, por ende, un pago por incentivos mayores. Por ello, el diseño del esquema de incentivos de los gerentes de los FC debe estar alineado con los de los inversionistas. Si un gerente de FC adopta una estrategia de comprar y vender constantemente, la frecuencia de sus operaciones puede ocasionar pagos de impuestos mucho más elevados que en el caso de una estrategia pasiva, es decir, de comprar y mantener. Este aumento de los impuestos reduce el rendimiento total que un inversionista puede recibir por su inversión, manteniendo todo lo demás en igualdad de condiciones.

La mayoría de los FC emplean el apalancamiento y la venta en corto con el fin de generar beneficios o como parte de sus políticas de cobertura contra el riesgo de caída de los mercados. Ambas estrategias pudieran aumentar el nivel de riesgo para el inversionista cuando son empleadas para especular o si sus efectos potenciales no han sido medidos correctamente. Así, con las ventas en corto, un FC puede perder una cantidad ilimitada de dinero, mientras que el apalancamiento puede magnificar las pérdidas.

\section{Revisión de los elementos más importantes de la crisis financiera global 2008-2009}

Existen varios factores que ayudan a entender el surgimiento de la crisis financiera que se desata en septiembre de 2008; sin duda, su origen se remonta a comienzos de la primera década del siglo XXI, cuando se conjugó una serie de elementos que facilitó un alza extraordinaria en los precios de los inmuebles en los países desarrollados y una mala gestión de los créditos hipotecarios (Amonlirdman, Getmansky, Kurma y Lo, 2004; Garay y Ter Horst, 2009; Acharya y Richardson, 2009; Acharya, Philippon, Richardson y Roubini, 2009; Levitin y Watcher, 2015). Algunos de los principales factores que exacerbaron el incremento en el precio de los inmuebles de los países desarrollados desde comienzos de siglo fueron: a) las bajas tasas de interés y disminución de los montos iniciales en el otorgamiento de créditos hipotecarios; b) los beneficios fiscales que incentivaron la adquisición de bienes raíces, y c) el optimismo desmedido que sobre el mercado inmobiliario existía en los inversionistas del momento.

El escenario descrito estimuló el endeudamiento hipotecario de las familias en estos países, que en muchos casos asumieron compromisos que no podrían honrar ante una disminución del precio de 
sus inmuebles o ante un incremento de las tasas de interés, incluso si este último fuese pequeño. Estos créditos hipotecarios de alto riesgo fueron conocidos como sub-prime. Más grave fue el hecho de que en Estados Unidos un gran porcentaje de los créditos otorgados a principio de la década se realizaron a tasas de interés variables; así, cuando en 2006 estas fueron liberadas, se ocasionó un encarecimiento sustancial de los pagos que debían realizarse (Mian y Sufi, 2011). Los principales afectados por este hecho fueron los integrantes del llamado segmento sub-prime, que pasaron de constituir el $8 \%$ del mercado hipotecario estadounidense en 2001, a 20\% en el 2006, al tiempo que pasaron a constituir el 75\% de la titularización de mercado en ese mismo año. El gráfico 1 muestra el espectacular aumento en el monto de las emisiones de títulos garantizados por hipotecas en los años que precedieron a la crisis y el posterior desplome durante la crisis.

Las entidades financieras y los prestamistas no bancarios (a quienes también se aprobó la entrada al mercado inmobiliario) estaban ansiosos por otorgar la mayor cantidad posible de créditos hipotecarios, sin detenerse mucho a evaluar la calidad crediticia de los prestatarios. Por otra parte, las evaluadoras crediticias sobrestimaron la calidad de estos préstamos hipotecarios y les atribuyeron calificaciones crediticias mucho más elevadas que las que correspondían. En poco tiempo, los créditos sub-prime llegaron a representar un volumen considerable de los balances de la banca estadounidense, lo cual llevó a muchas de estas instituciones a colocar estos títulos financieros en fondos de inversión paralelos, y a luego empaquetarlos junto con otros títulos, creando instrumentos financieros altamente sofisticados y con elevada calificación crediticia (en muchos casos "AAA"), que eran vendidos en los mercados financieros internacionales. Así, inversionistas alrededor del mundo, atraídos por la atractiva rentabilidad que estos papeles ofrecían y por su aparente solvencia crediticia, se dedicaron a la compra masiva de estos instrumentos.

La Reserva Federal de Estados Unidos también desempeñó un rol importante al exacerbar algunas de las distorsiones que desembocaron en la crisis financiera de 2008-2009 (Ivashina y Scharfstein, 2010). Para algunos economistas, al haber mantenido las tasas de interés a niveles históricamente bajos desde comienzos de siglo (a pesar de los aumentos de 2004 y 2006), en respuesta al desplome de las acciones tecnológicas ocurrido entre 2000 y 2002, la Reserva Federal potenció la aparición de una burbuja

Gráfico 1.

Emisiones de títulos garantizados por hipotecas, 1998-2009, en Estados Unidos*

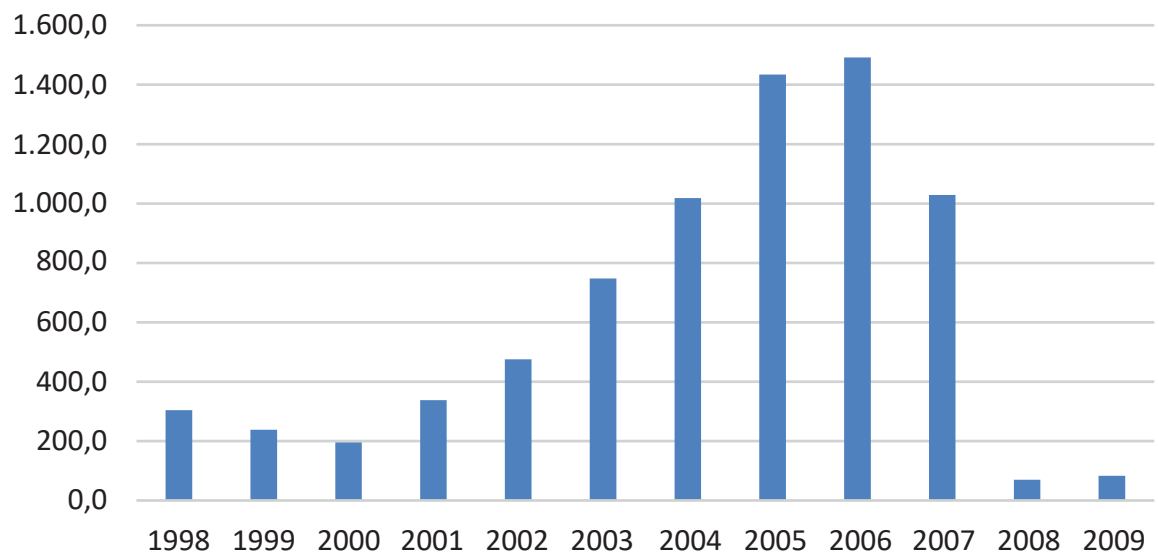

Nota: no incluye las emisiones realizadas por agencias tales como FNMAE, GNMAE y FredyMAC. Cifras en miles de millones de USD.

Fuente: Sifma. 
en los precios de los inmuebles y un crecimiento exponencial del mercado de hipotecas, factores que constituyen dos de las razones principales que explican la crisis de 2008-2009, una vez que los precios de los inmuebles comenzaron a caer.

A lo anterior se unió la idea que había en el mercado de que la Reserva Federal auxiliaría a aquellas instituciones financieras que pudieran llegar a tener problemas (debido a la mentalidad del "too big to fail" que había quedado en el ambiente después de la intervención del FC Long Term Capital Management en 1998). De ese modo, se planteaba un problema grave de riesgo moral en las instituciones financieras (Covitz, Liang y Suárez, 2009).

El alza sostenida de los precios de los inmuebles no se pudo mantener indefinidamente, lo cual ocasionó que, para septiembre de 2008 , estos cayeran en promedio un $20 \%$ con respecto a los precios alcanzados apenas dos años antes. Este hecho derivó en que una gran cantidad de prestatarios se vieran incapaces de cubrir sus deudas, ya que en muchos casos estas excedían al valor de mercado de sus inmuebles. Se desencadenaron así graves problemas en instituciones financieras alrededor del mundo, las cuales habían adquirido títulos emitidos sobre esos créditos hipotecarios (en inglés, mortgage backed securities). En Estados Unidos, todo lo anterior causó un mayor descenso en los precios de los inmuebles, la quiebra de una serie de instituciones financieras (grandes y pequeñas), la caída de los precios de las acciones de empresas en los mercados de capitales en el ámbito mundial (la mayor caída en ochenta años), la caída en el consumo privado y la bancarrota de importantes sectores empresariales. El desempleo y la caída de las economías fueron resultado de esta crisis.

\section{Efecto de la crisis sobre los fondos de cobertura y sobre los fondos de fondos de cobertura}

La crisis de 2008-2009 fue precipitada por la quiebra del banco de inversión Lehman Brothers, y afectó negativamente a muchos FC. Lehman Brothers tenía en sus balances altas sumas invertidas en hipotecas titularizadas, las cuales, al descender de

Gráfico 2 .

Índice de precios de las acciones en el ámbito mundial, diciembre de 1999 a diciembre de 2009*

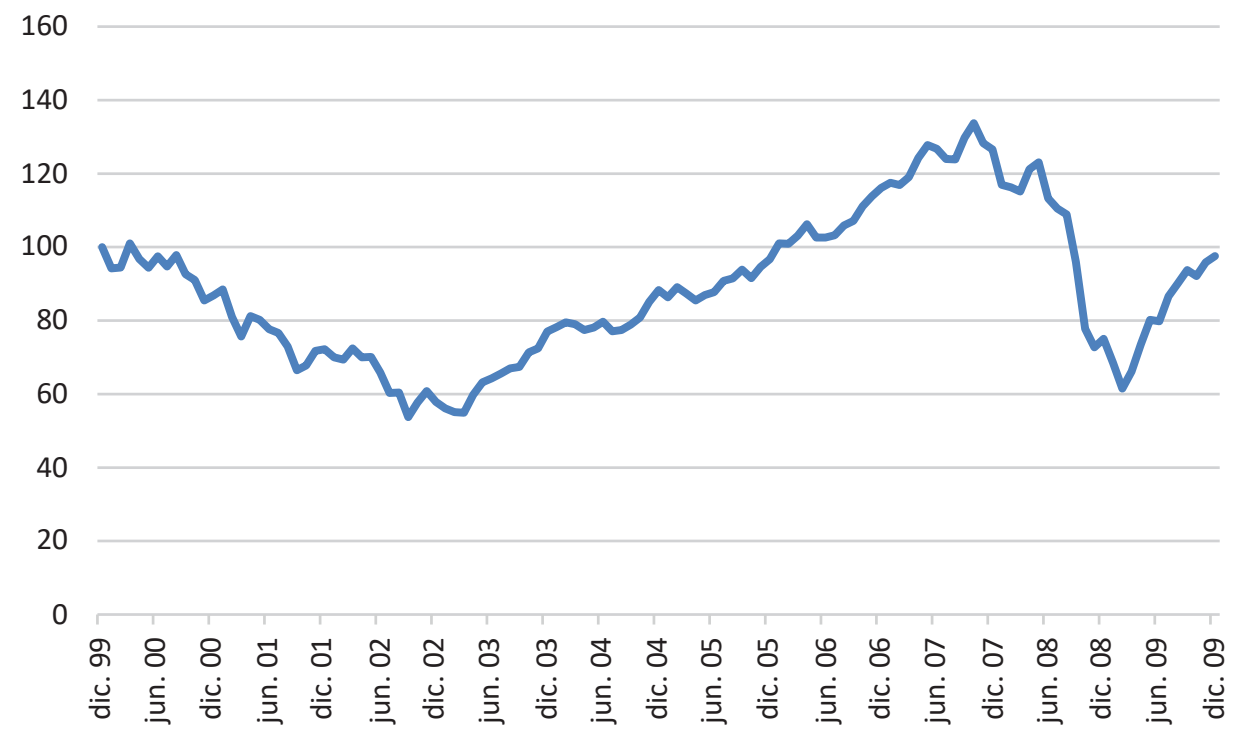

Nota: Índice de precios medido con el índice de rendimientos totales MSCI (Morgan Stanley Capital Index) mundial. Datos en USD; diciembre de $1999=\$ 100$.

Fuente: Datastream. 
valor, pusieron en aprietos a esta histórica institución. La quiebra de Lehman Brothers en septiembre de 2008 fue desastrosa para muchos FC, debido a que este banco de inversión era su principal corredor primario (prime broker). La quiebra de esta institución implicó que estos FC ya no contaban con su corredor primario para poder realizar ventas en corto, solicitar préstamos y realizar operaciones de compra y venta de valores.

A lo anterior se unió el efecto que el desplome abrupto de las bolsas de valores en el ámbito mundial (gráfico 2) causó sobre los valores de las carteras de los FC. Ello condujo, en no pocos casos, al cierre del FC respectivo. Ante las amortizaciones, las restricciones a la realización de ventas en corto de acciones de algunas empresas, los aumentos en los costos de financiamiento y la imposibilidad de apalancarse, muchos FC no pudieron mantener sus estrategias de inversión. Así, la gravedad de la crisis financiera global de 2008-2009 — que ha sido catalogada como la peor crisis financiera desde la Gran Depresión de los años treinta- representa un evento idóneo para determinar las características de los FFC que fueron capaces de sobrevivir a semejante crisis.

Existe una gran coincidencia entre las crisis financieras de 1998 y de 2008. Ambas crisis se precipitaron por la quiebra de dos prominentes instituciones financieras: el FC Long-Term Capital Managment (LTCM) en 1998 y el banco de inversión Lehman Brothers en 2008. LTCM fue uno de los más grandes FC, y Lehman era uno de los más grandes bancos de inversión. Ambas instituciones eran contrapartes de una gran cantidad de acuerdos de derivados y swaps. Lehman Brothers era contraparte para muchos FC en contratos de derivados, swaps y apalancamiento; los bonos de Lehman eran también utilizados como colaterales por los FC. La bancarrota de Lehman ocasionó un aumento en el riesgo de contraparte, las limitaciones para fondos de los fondos de cobertura y para mantener sus posiciones de arbitraje, además del cese de las operaciones de intermediación financiera.

Ambos eventos llevaron a un congelamiento de los mercados de crédito y tuvieron un severo impacto global. Cuando Lehman Brothers se declaró en bancarrota, inmediatamente quebró su primera agencia de corretaje en el Reino Unido. Esto condujo a pérdidas masivas en muchos FC, debido a la desaparición de sus valores, los cuales habían sido colocados como colaterales. Además, en el peor momento de la crisis, se congelaron las actividades interbancarias debido a que ningún banco confiaba en la solvencia financiera del otro y la actividad de intermediación financiera llegó a estar en riesgo de colapsar completamente (Acharya et al., 2009).

Aunque muchos FC utilizan estrategias de cobertura en las que los movimientos del mercado están parcial o completamente contrarrestados, estos fondos están inherentemente expuestos a otros riesgos, tales como el riesgo de volatilidad, de crédito y de liquidez, los cuales se exacerbaron durante la crisis a niveles tales que muy pocos gerentes de fondos habían contemplado como potencialmente factibles en sus modelos. Además, las posiciones de muchos de los FC no estaban del todo cubiertas, por lo que los efectos de la crisis, con desplomes en los precios de la mayoría de los títulos, aunada a una situación de aguda iliquidez para valores tales como las hipotecas titularizadas, en medio de carteras altamente apalancadas, representó una tormenta perfecta para muchos FC, con el consecuente efecto negativo sobre los FFC.

\section{Literatura sobre los modelos ABS y Probit para predecir la probabilidad de sobrevivencia}

En esta sección se repasa brevemente la literatura relacionada con los modelos ABS y Probit para predecir la probabilidad de sobrevivencia de FC. Varios modelos han sido propuestos para evaluar y analizar los determinantes del desempeño y el riesgo de los FC, tomando como muestra diferentes periodos y tipos de estrategias seguidas por estos fondos. Entre ellos se encuentra el modelo Asset-Based Style Factors Model (ABS) o modelo de factores de estilo basados en activos. Los factores ABS son claves para la construcción de un portafolio y para 
la evaluación del desempeño ajustado a riesgo de los fondos. Si bien este modelo no es utilizado en el análisis empírico que se desarrolla más adelante, es bueno repasarlo aquí debido a que ha sido ampliamente utilizado en los estudios sobre FC, incluidos algunos estudios sobre el comportamiento de los FC en tiempos de crisis.

Fung y Hsieh (2004) proponen una extensión del modelo de Sharpe (1992), el cual se comentará brevemente más adelante; utilizaron un modelo de factores múltiples para examinar el desempeño de los FC, estimando el retorno de rendimiento y el riesgo. El modelo sigue el estándar de rendimiento ajustado a riesgo.

La ecuación por estimar en el modelo ABS tiene la siguiente especificación:

$$
R_{t}=\propto+\sum_{k} b_{k} S F_{k, t}+u_{t}
$$

Donde: $\left\{R_{t}\right\}$ es el retorno del fondo en el tiempo, el término $\left\{S_{k, t}\right\}$ contiene las variables, las cuales son factores de estilo, $\left\{b_{k}\right\}$ son los pesos factoriales de las variables (betas de cada factor) y $\left\{u_{t}\right\}$ es el residuo en cada unidad de tiempo.

Sharpe (1992) denomina a esta ecuación como un modelo factorial, donde usa las siguientes clases de activos: acciones de Estados Unidos y del resto del mundo (medidas por el índice $\mathrm{MSCl}$ ), y acciones de mercados emergentes (medidas por el índice IFC). Hay dos clases de bonos: del gobierno Estados Unidos y de gobiernos de otros países.

Fung y Hsieh (2004) determinan, de manera similar al modelo de Sharpe (1992), ocho clases de activos para el modelo de factores; establecen que un modelo lineal de ocho factores es capaz de proporcionar estimaciones satisfactorias de combinaciones de activos para una muestra grande de FC.

Billio, Getmansky y Pelizzon (2009) estudiaron los efectos de las crisis financieras en el riesgo de los FC; encuentran que en periodos de crisis aumentaba la volatilidad y la correlación promedio de los retornos de sus estrategias, y que esto se debía, en parte, a su exposición tanto a factores de riesgo sistémicos (liquidez, crédito y volatilidad) como a factores de riesgo latentes (idiosincrásico). Encontraron también que, en épocas de crisis y periodos de alta volatilidad, los gerentes de FC reducían su exposición al mercado. Más recientemente, Getmansky, Lee y Lo (2015) argumentan que la crisis financiera tuvo un efecto negativo sin precedentes sobre la industria de los FC, al punto de que el $70 \%$ de ellos sufrieron rendimientos negativos en 2008 y un número récord de fondos desaparecieron.

Xu, Liu y Loviscek (2011) emplean un modelo multifactorial para analizar los riesgos, las características y el desempeño de los FC antes de la crisis, y analizan si esas variables pudieran ser utilizadas para predecir el desempeño de los FC durante la crisis financiera global de 2008-2009. El modelo sigue el marco de desempeño basado en riesgo, tales como los propuestos por Fung y Hsieh (2002), Fung, Hsieh, Ramadorai y Naik (2008), Bollen y Do Pool (2009), Bollen y Whaley (2009) y, más recientemente, Garay, Molina y Ter Horst (2016).

El modelo de Xu, Liu y Loviscek (2011) se compone de los cinco factores de riesgo de seguimiento de tendencias propuestas por Fung y Hsieh (2002): un portafolio de opciones obtenido bajo un straddle de bonos (POB), un portafolio de opciones obtenido bajo un straddle de monedas (POM), un portafolio de opciones obtenido bajo un straddle de commodity (POC), la tasa de interés de corto plazo simulada (TIC) y el índice de acciones (IAS) simulado. A su vez, el modelo incluye otros cinco factores lineales: los tres factores de Fama y French (1992 y 1993): exceso de retorno sobre mercado accionario, tamaño del factor y el valor del factor; el diferencial de crédito medido por el cambio entre los bonos Moody's Baa menos el bono del tesoro de Estados Unidos a diez años, y el diferencial entre diez años y tres meses de bonos del tesoro de Estados Unidos.

Xu, Liu y Loviscek (2011) utilizan datos mensuales desde enero de 2005 hasta de enero de 2007, establecido por los autores como el periodo precrisis, para la estimación de los retornos y de los rendimientos como medida de riesgo de los FC. 
A continuación, estos autores emplean un modelo Probit para predecir la probabilidad de sobrevivencia de un FC. La variable dependiente es Attrition, lo que equivale a $O$ si un fondo se "inactiva" (deja de reportar a las bases de datos de FC) a partir de la crisis, entre febrero de 2007 y marzo de 2009, y 1 si todavía estaba activo a marzo de 2009. Xu, Liu y Loviscek (2011) consideraron varios factores o variables microeconómicas de los FFC, de las cuales las siguientes seis fueron significativas: edad del FC, su tamaño, promedio de retorno mensual, desviación estándar de los rendimientos, auditoría y apalancamiento, medidas como sigue:

- Edad FC (en meses) desde que se creó el FC hasta marzo de 2009 (para los fondos activos) o el último mes de reporte (para los fondos inactivos).

- Tamaño: valor promedio de los activos del fondo, en dólares de Estados Unidos, durante el periodo precrisis (es decir, entre enero de 2005 y enero de 2007).

- Promedio de retorno mensual, durante el periodo precrisis, enero de 2005 hasta enero de 2007.

- Desviación estándar de sus retornos mensuales, durante el periodo precrisis, enero de 2005 hasta enero de 2007.

- Auditoría: es igual a 1 si el fondo es auditado anualmente y 0 en caso contrario.

- Apalancamiento: es igual a 1 si el fondo utiliza apalancamiento y 0 en caso contrario.

La principal innovación del presente trabajo consiste en la aplicación del modelo Probit para intentar explicar la probabilidad de que los FFC (en lugar de los FC, ya estudiados por otros autores) hubieran permanecido activos después de la crisis. Además, en el modelo Probit propuesto aquí se incluyen algunas variables que no habían sido contempladas en el trabajo de $\mathrm{Xu}$, Liu y Loviscek (2011), a saber: autocorrelación de los rendimientos, curtosis y sesgo de la distribución, frecuencia de redención, periodo para anuncio de redención, porcentaje de incentivo, comisión por gerencia del fondo e inversión mínima en el fondo.

\section{HIPÓTESIS Y VARIABLES}

En esta parte del trabajo se enuncia la hipótesis y se describen la variable dependiente y las variables independientes.

\section{Hipótesis}

La probabilidad de sobrevivencia de los FFC durante la crisis financiera global 2008-2009 puede ser explicada por el comportamiento de una serie de variables microeconómicas de los fondos, las cuales se listan a continuación.

\section{Variable dependiente}

Se definió como variable dependiente a la variable dicotómica Activo, la cual define la actividad (valor igual a 1) o inactividad (valor igual a 0 ) de un FFC, tomando el criterio de CISDM, según el cual la inactividad ocurre cuando se registran cuatro meses continuos durante los que el FFC respectivo no reporta los rendimientos obtenidos y los activos gestionados a la base de datos CISDM, para los meses previos a septiembre de 2009 .

\section{Variables independientes}

Se consideraron catorce variables independientes, las cuales están representadas por las siguientes características microeconómicas de los FFC, obtenidas a través de la base de datos de CISDM:

1. Autocorrelación de los rendimientos. Corresponde a la autocorrelación de los rendimientos mensuales del FFC, medida durante los 24 meses previos a la crisis.

2. Curtosis. Es el coeficiente de curtosis de los rendimientos mensuales del FFC, medido durante los 24 meses previos a la crisis.

3. Edad del fondo. Es la edad reportada por el FFC, en meses, durante el mes previo a la crisis.

4. Frecuencia de redención. Está determinada por la frecuencia temporal con la cual los 
inversionistas pueden retirar dinero del fondo; está medida en meses.

5. Periodo para el anuncio de redención. Corresponde al periodo de antelación con el cual un inversionista puede informar su intención de retirar dinero del fondo.

6. El fondo usa financiamiento. Es una variable dicotómica, la cual se creó tomando como base el mes previo a la crisis, asignándosele un valor igual a 1 a aquellos FFC que usaban financiamiento, y un valor igual a 0 a aquellos que no se apalancaban.

7. Porcentaje de incentivo. Es el porcentaje calculado sobre las ganancias que arrojó el FFC por encima de un determinado benchmark en un periodo, que, por lo general, es de un año, otorgado al gerente del fondo como incentivo por su desempeño.

8. Comisión por gerencia del fondo. Es un porcentaje fijo aplicado sobre el valor total de los activos gestionados por el FFC, el cual es destinado a cubrir los costos de manejo del fondo.

9. Inversión mínima. Corresponde al monto mínimo de inversión exigido por el FFC.

10. Sesgo. Corresponde al sesgo de los rendimientos del FFC, que se calcula utilizando los 24 meses previos a la crisis.

11. Auditado. Es una variable dicotómica, que se creó tomando como base el mes previo a la crisis, asignándosele un valor igual 1 a aquellos FFC que contaban con una compañía auditora y un valor igual a 0 a aquellos que no.

12. Rendimiento promedio antes de la crisis. Corresponde al rendimiento promedio del FFC, que se calcula utilizando el promedio de los 24 meses previos a la crisis.

13. Desviación de los rendimientos. Corresponde a la desviación estándar de los rendimientos de los FFC, que se calcula utilizando los 24 meses previos a la crisis.

14. Tamaño. Esta variable fue considerada calculando el logaritmo neperiano del valor total de los activos gestionados por el fondo durante el mes previo a la crisis.

\section{DATOS Y METODOLOGÍA}

Para esta investigación se utilizó la base de datos CISDM-Morningstar, la cual fue gentilmente suministrada por el Center for International Securities and Derivatives Markets (CISDM) de la Universidad de Massachusetts, Amherst. El estudio se basó en la elaboración de un análisis de corte transversal de las estadísticas descriptivas de los FFC y en la construcción de un modelo de regresión Probit, estimado a través del paquete econométrico SAS, a fin de determinar qué variables microeconómicas desempeñaron un papel significativo en la condición de actividad o inactividad de los FFC, como resultados de la crisis financiera global de 20082009. Los pasos seguidos para la elaboración del análisis y la construcción del modelo de regresión fueron los siguientes:

1. Definición del periodo de crisis financiera. Con la finalidad de acotar el periodo para la realización del estudio, se definió como periodo de crisis al lapso septiembre de 2008 a junio de 2009. Se escogió el mes de septiembre de 2008 como comienzo de la crisis debido a que, como se comentó, en ese mes Lehman Brothers se fue a la bancarrota, causando así una serie de disrupciones tanto en los mercados financieros como en los FC para los cuales ese banco de inversión era su corredor primario.

2. Selección del universo de FFC. Como punto inicial, se seleccionaron únicamente para la elaboración del estudio a aquellos FFC que reportaron tanto rendimientos como valor de los activos gestionados, el mes previo a la crisis, es decir, agosto de 2008. Esto con la finalidad de no incluir a aquellos FFC que se encontraban inactivos antes de la crisis.

3. Cálculo de las variables microeconómicas de los FFC. Utilizando los FFC seleccionados, se procedió a calcular las siguientes variables para los rendimientos mensuales reportados los 24 meses previos a la crisis: el valor promedio, la desviación estándar, la autocorrelación, el coeficiente de curtosis y el sesgo. Adicionalmente, se creó una variable llamada Auditado, la cual 
tomó el valor 1 para aquellos FFC que se encontraban auditados el mes previo a la crisis, y 0 para los que no.

4. Categorización de FFC en activos e inactivos. Consistió en la creación de dos grupos de FFC: activos e inactivos, con la finalidad de apreciar diferencias en las características y estadísticas descriptivas de estos dos grupos. Dicha separación se realizó tomando como referencia la variable Active de la base de datos CISDM, la cual toma el valor de 1 para aquellos fondos que se encontraban activos en agosto de 2009, y 0 para aquellos FFC que se encontraban inactivos en la misma fecha.

5. Cálculo de estadísticas descriptivas. Utilizando los FFC seleccionados, se calculó el valor de la media, desviación estándar, mediana, mínimo y máximo para los grupos de FFC, activos e inactivos, de forma conjunta y separada. Esto se realizó con el objeto de observar las diferencias que pudieran existir entre las distintas variables seleccionadas para el grupo de FFC activos e inactivos, y de esa forma inferir cuales podían ser los resultados que podrían obtenerse como producto del modelo de regresión Probit.

6. Regresión Probit. Se construyó un modelo de regresión Probit, siendo la variable dependiente si el FFC se encontraba activo o inactivo, es decir, una variable dicotómica, e introduciendo las catorce variables independientes descritas. De esta forma, la expresión para el modelo de regresión quedó planteada de la siguiente forma:

$$
\mathrm{Y}=\beta_{0}+\beta_{1} X_{1}+\beta_{2} X_{2}+\beta_{3} X_{3}+\beta_{4} X_{4}+\beta_{5} X_{5}+
$$

$\beta_{6} X_{6}+\beta_{7} X_{7}+\beta_{8} X_{8}+\beta_{9} X_{9}+\beta_{10} X_{10}+\beta_{11} X_{11}+\beta 1_{2} X 1_{2}+$ $\beta_{13} X_{13}+\beta_{14} X_{14}+\beta_{14} X_{14}$

Donde $Y$ es la variable dependiente, $\beta_{o}$ es la constante de la regresión, las $\beta_{i}$ son los coeficientes estimados de la regresión y las $X_{i}$ son las variables independientes explicadas anteriormente.

7. Contraste de prueba de hipótesis. A fin de evaluar la significancia que tuvieron cada una de las variables independientes, se realizó la siguiente prueba de hipótesis con un nivel de significancia del $5 \%$ :

Ho: el coeficiente $\beta_{\mathrm{i}}$ de la variable $\mathrm{X}_{\mathrm{i}}$ es igual a cero $\rightarrow \beta_{i}=0$

Ha: el coeficiente $\beta_{\mathrm{i}}$ de la variable $\mathrm{X}_{\mathrm{i}}$ es distinto de cero $\rightarrow \beta_{\mathrm{i}} \neq 0$

8. Incidencia de las variables independientes. Adicionalmente, como resultado de la regresión, se obtuvieron los estimados de los coeficientes $\beta_{i}$ con sus respectivos signos. Esto sirvió para determinar si la influencia de la variable independiente causaba que el valor de la variable dependiente tendiese a 1 o a 0 , es decir, a que el FFC estuviese activo o inactivo.

\section{ANÁLISIS Y RESULTADOS}

Con base en el análisis de los datos, pudieron apreciarse ciertas características de los FFC en relación con su evolución en lo que respecta al monto total de activos gestionados y al número de fondos existentes, rendimientos registrados antes y durante la crisis, estrategias seguidas y las correspondientes estadísticas descriptivas. A continuación, se ofrece el análisis correspondiente.

\section{Activos gestionados y número de fondos}

En el gráfico 3 se aprecia la evolución en el monto total de los activos gestionados por los FFC durante el periodo 1995-2009. Esta evolución está caracterizada por subidas y bajadas en el total de los activos; se observa además una tendencia creciente hacia 2007 y 2008, seguida por una caída pronunciada en 2009, coincidente con la crisis financiera global.

Como puede apreciarse en el gráfico 4, el monto total de activos gestionados por los FFC existentes antes de la crisis financiera se encontraba alrededor de los 500.000 millones de dólares. Asimismo, puede apreciarse la evolución de este monto a lo largo del periodo estudiado, es decir, entre septiembre de 2008 y junio de 2009. 
Activos gestionados por FFC MMUS\$ (1995-2009)

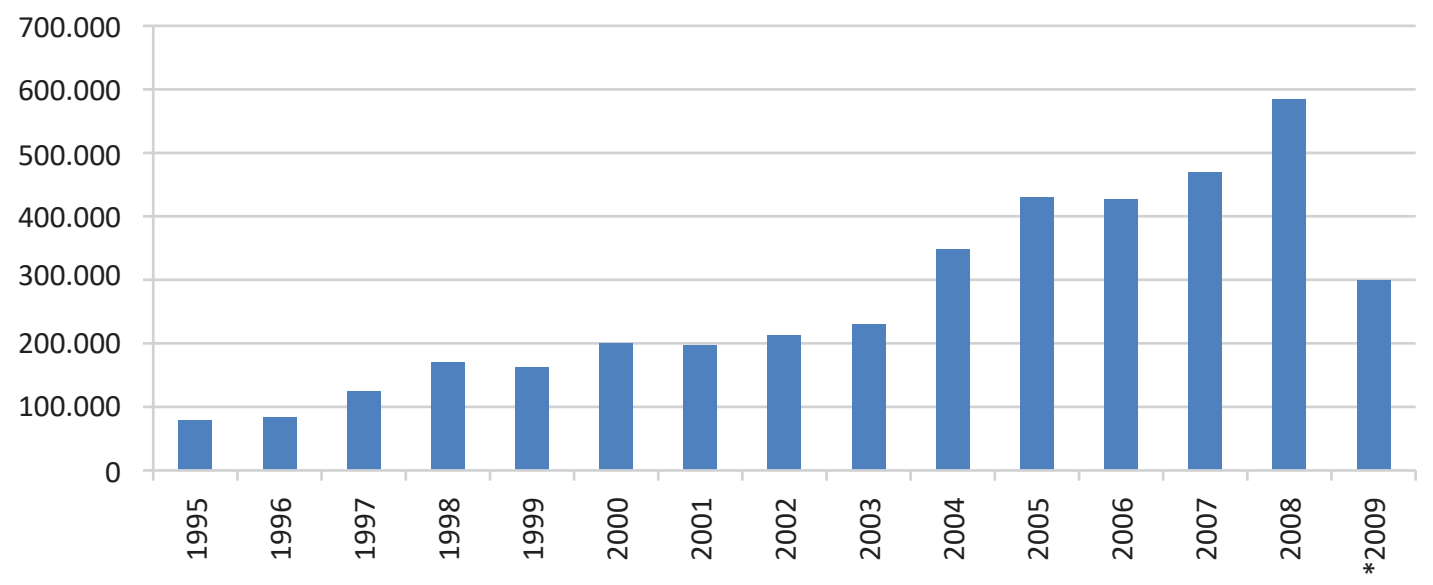

Fuente: Base de datos CISDM.

Gráfico 4.

Activos gestionados por FFC MMUS\$ (enero de 2008 a julio de 2009)

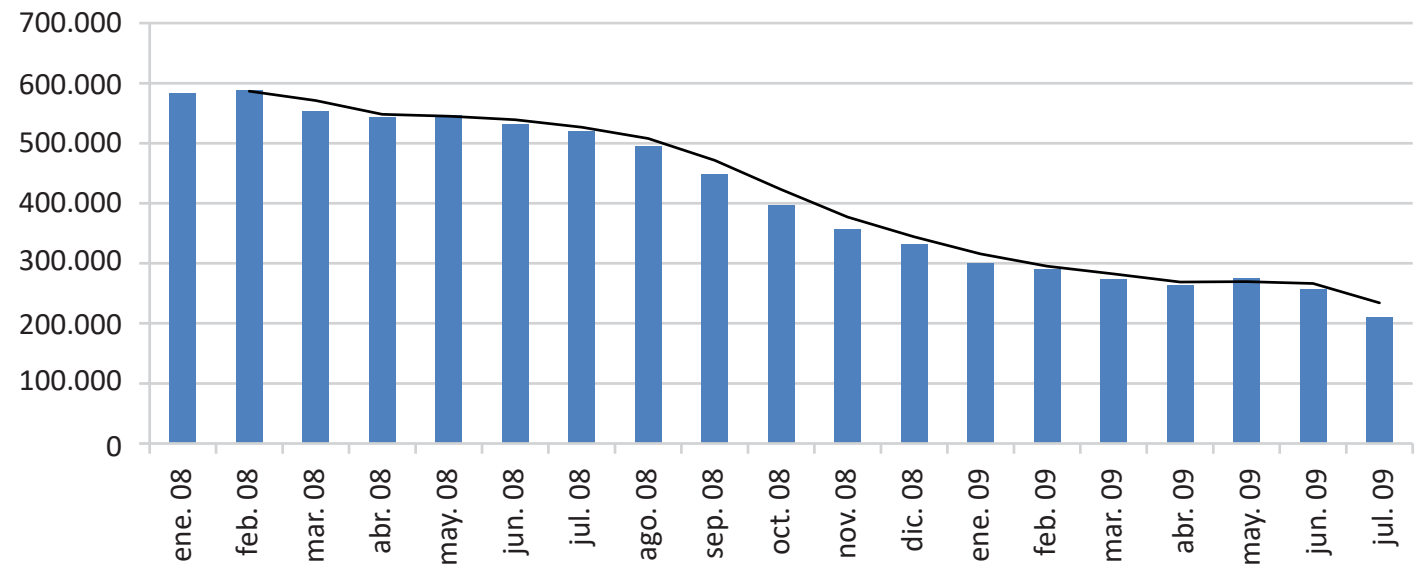

Fuente: Base de datos CISDM.

En relación con el número de $\mathrm{FFC}$ existentes, en el gráfico 5 se aprecia cómo en agosto de 2008 el número ascendía a 1057 fondos; sin embargo, para agosto de 2009, este número había decrecido en un $25 \%$, alcanzando los 797 fondos. 
Número de fondos de fondos activos (agosto de 2008 y agosto de 2009)

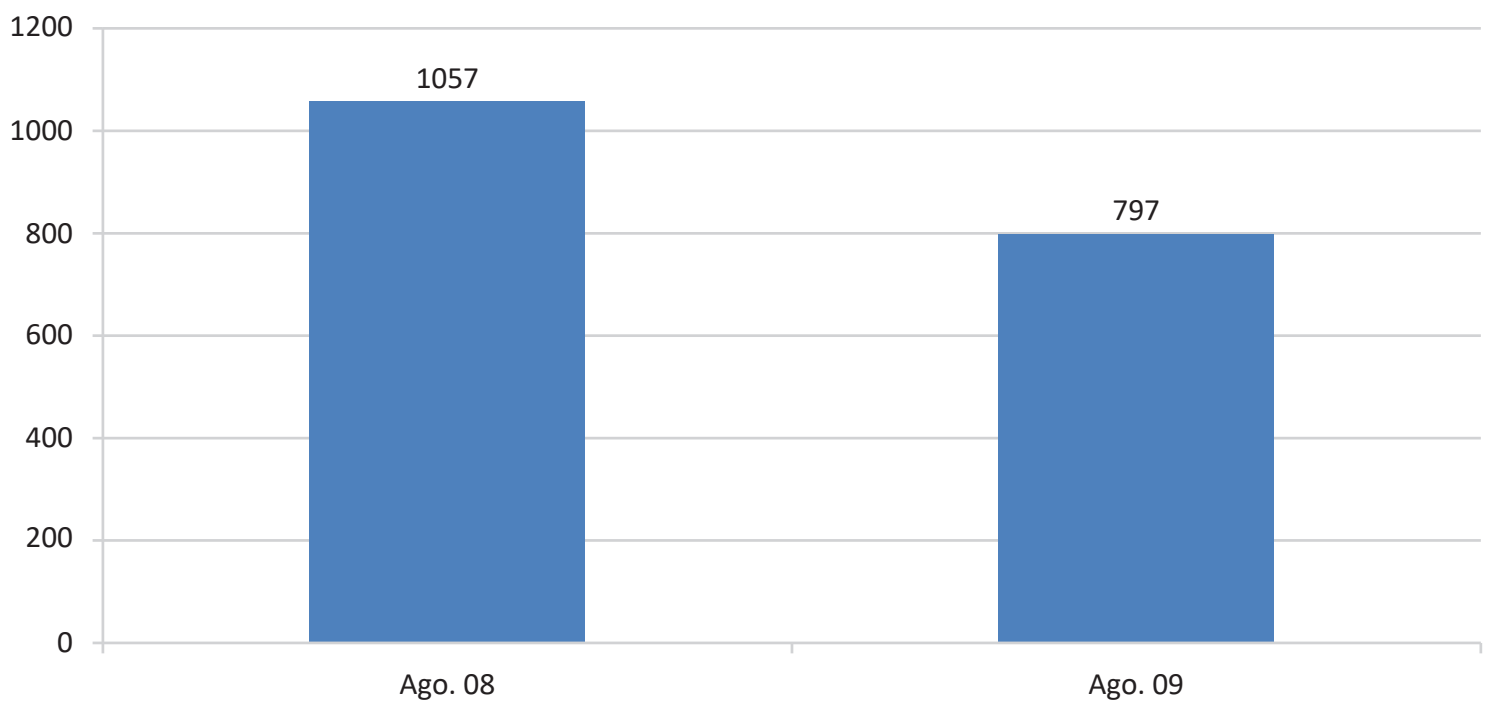

Fuente: Base de datos CISDM

\section{Rendimientos}

Como puede apreciarse en el gráfico 6, previo al estallido de la crisis financiera en septiembre de 2008, existió una escasa diferencia entre la evolución de los rendimientos mensuales para el grupo de FFC que se encontraban activos después de la crisis, en relación con los que pasaron a estar inactivos después de la crisis. En todo caso, a pesar de que los rendimientos de ambos grupos se movieron con la misma tendencia, existió una ligera diferencia a favor de los FFC activos.

\section{Estrategia}

Respecto a la estrategia que seguían los FFC para el mes previo a la crisis, el gráfico 7 muestra que la mayoría de los FFC implementaban una multiestrategia (el $73 \%$ de los FFC), seguida por fondos de una sola estrategia, los cuales representaban el $19 \%$ del universo de fondos incluidos en la base de datos para esta fecha.

Sin embargo, si se observan los dos grupos de FFC por separado, activos e inactivos, se puede apreciar ciertas diferencias, aunque no muy marcadas, en las estrategias que seguían estos dos grupos de fondos antes de la crisis. Como puede observarse en el gráfico 8 , los fondos que seguían una multiestrategia representaban un $74 \%$ de los fondos activos, mientras que para los inactivos esta estrategia representaba un 69\%; esta diferencia porcentual para estos últimos se distribuía en la estrategia única. Se pudiera argumentar que los FFC multiestrategia, al invertir en varias estrategias de FC al mismo tiempo (como indica su nombre), debían estar más diversificados que los FFC que seguían una sola estrategia, por lo cual un mayor porcentaje de estos logró sobrevivir a la crisis (pasando así a representar un $74 \%$ de los FFC después de la crisis desde un menor $69 \%$ antes de la crisis).

\section{Estadísticas descriptivas de las principales variables microeconómicas de los FFC}

Al analizar la tabla 1, se puede notar la existencia de una gran variabilidad (desviación estándar) en los datos para cada una de las variables, aunque en esta tabla los FFC no fueron separados por activos e inactivos. Sin embargo, a fin de entender en 
Rendimientos mensuales promedio de los FFC (septiembre de 2006 a agosto de 2008)

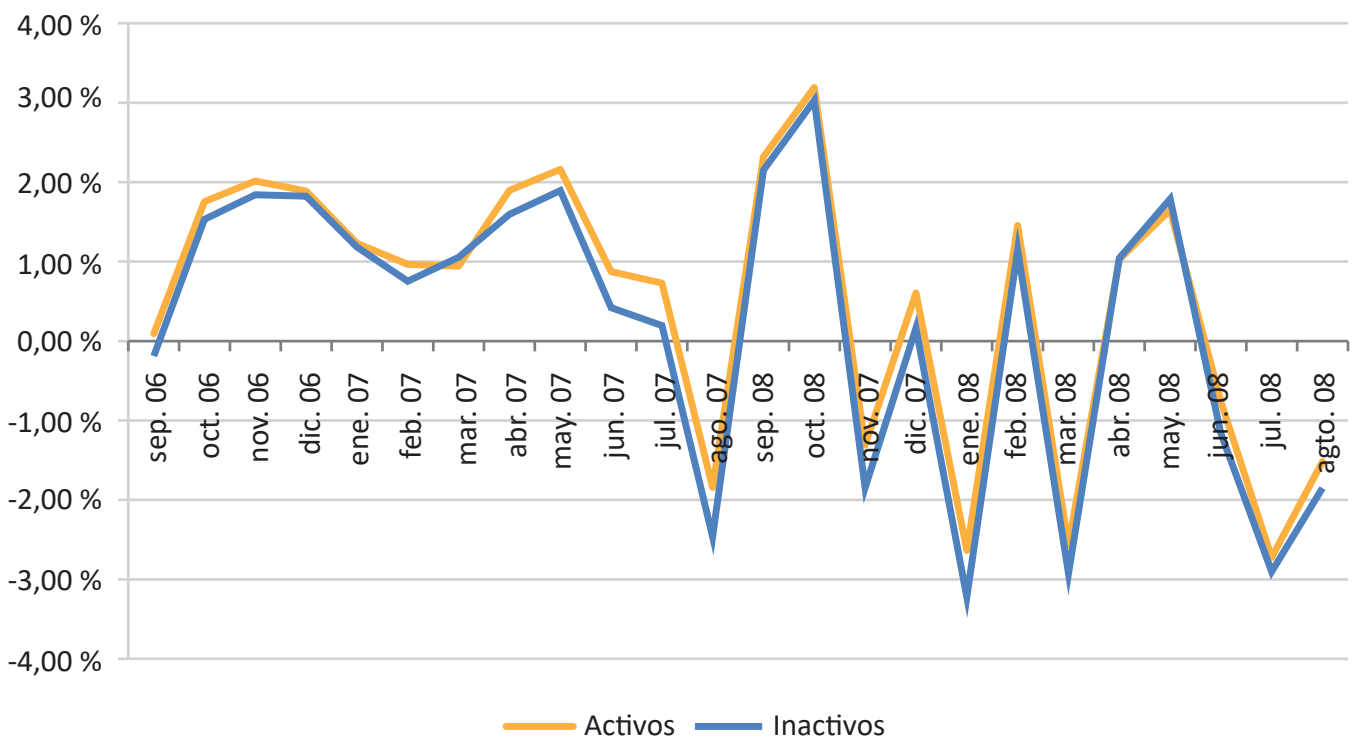

Fuente: Base de datos CISDM.

Gráfico 7.

Distribución porcentual de las estrategias seguidas por los FFC en agosto de 2008

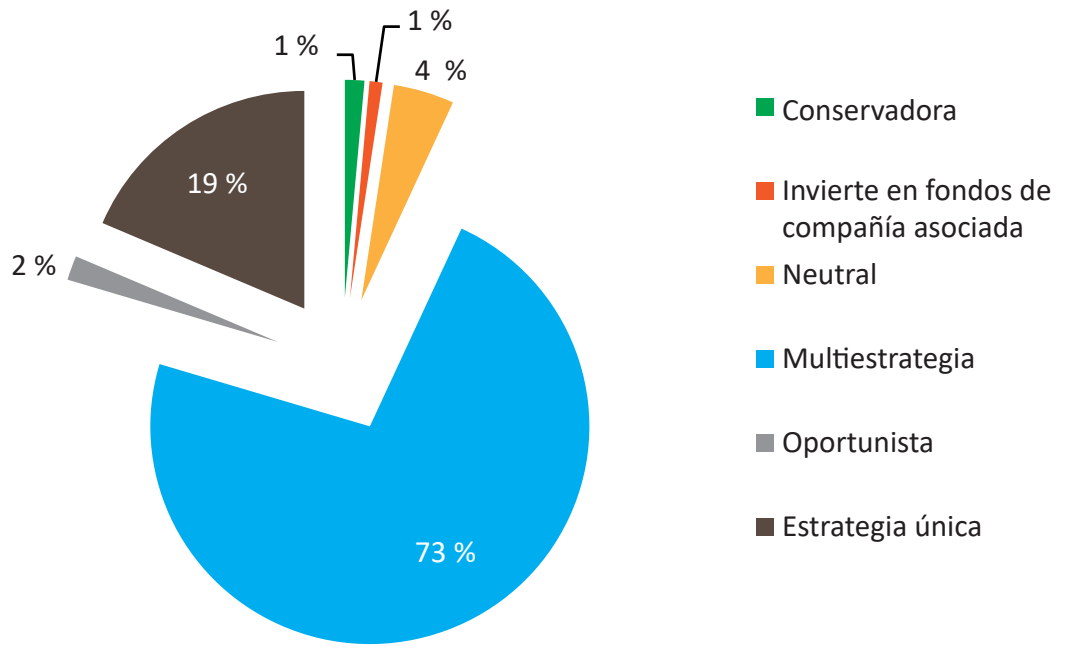

Fuente: Base de datos CISDM.

más detalle algunos de los atributos de los FFC, se procedió a analizar sus estadísticas y características, separándolos en aquellos FFC que se encontraban activos de aquellos que pasaron a estar inactivos después del estallido de la crisis, y analizando los valores que registraron las variables en el mes previo a la crisis. Así, en la tabla 2 se recogen los $p$-valores asociados a la prueba $t$ de Student para 
Distribución porcentual de las estrategias seguidas por los FFC, activos e inactivos, en agosto de 2008

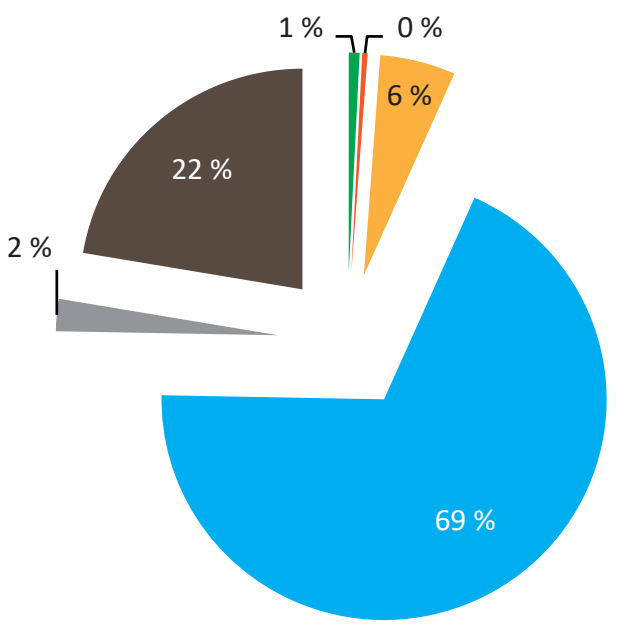

Fondos

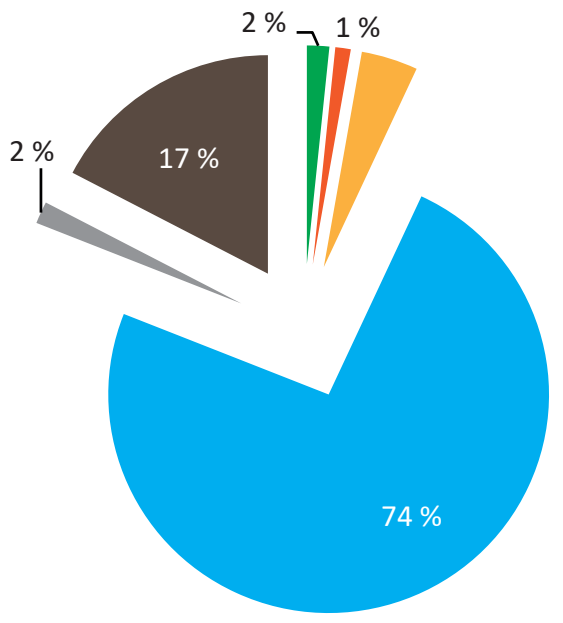

Fondos activos
Conservadora

- Invierte en fondos de compañía asociada

Neutral

Multiestrategia

Oportunista

- Estrategia única

Fuente: Base de datos CISDM

Tabla 1 .

Estadística descriptiva de las variables microeconómicas de todos los FFC, medidas en agosto de 2008

\begin{tabular}{|c|c|c|c|c|c|c|}
\hline Variable & Media & $\begin{array}{l}\text { Desviación } \\
\text { estándar }\end{array}$ & Mediana & Mínimo & Máximo & $\begin{array}{l}\text { Número de } \\
\text { observaciones }\end{array}$ \\
\hline $\begin{array}{l}\text { Autocorrelación de } \\
\text { los rendimientos }\end{array}$ & 0,076 & 0,207 & 0,072 & $-1,000$ & 1,000 & 1.037 \\
\hline Curtosis & 0,319 & 1,929 & $-0,155$ & $-5,146$ & 18,423 & 1.027 \\
\hline Edad del fondo (meses) & 73,66 & 48,48 & 67,00 & 0,00 & 164,00 & 1.057 \\
\hline Frecuencia de redención & 2,85 & 3,05 & 3,00 & 0,00 & 12,00 & 1.057 \\
\hline $\begin{array}{l}\text { Periodo para anuncio } \\
\text { de redención }\end{array}$ & 58,18 & 28,02 & 60,00 & 0,00 & 190,00 & 970 \\
\hline $\begin{array}{l}\text { El fondo usa financiamiento } \\
\qquad\left(s^{\prime}=1, \text { no }=0\right)\end{array}$ & 0,397 & 0,490 & 0,000 & 0,000 & 1,000 & 866 \\
\hline Porcentaje de incentivo & 10,42 & 6,66 & 10,00 & 0,00 & 30,00 & 969 \\
\hline $\begin{array}{l}\text { Comisión por gerencia } \\
\text { del fondo (\%) }\end{array}$ & 1,31 & 0,52 & 1,50 & 0,00 & 10,00 & 1.023 \\
\hline Inversión mínima (\$) & 1.658 .447 & 18.661 .891 & 250.000 & 0 & 500.000 .000 & 990 \\
\hline Sesgo & $-0,604$ & 0,621 & $-0,651$ & $-4,106$ & 2,656 & 1.040 \\
\hline $\begin{array}{c}\text { Auditado } \\
(\text { sí }=1, \text { no }=0)\end{array}$ & 0,817 & 0,387 & 1,000 & 0,000 & 1,000 & 1.057 \\
\hline $\begin{array}{c}\text { Rendimientos promedio } 24 \text { meses } \\
\text { antes de la crisis }\end{array}$ & 0,003 & 0,007 & 0,004 & $-0,051$ & 0,036 & 1.054 \\
\hline $\begin{array}{l}\text { Desviación de los rendimientos } \\
24 \text { meses previos }\end{array}$ & 0,023 & 0,012 & 0,020 & 0,001 & 0,099 & 1.047 \\
\hline Tamaño (millones de dólares) & 18,08 & 1,52 & 18,05 & 12,77 & 22,07 & 847 \\
\hline
\end{tabular}

Fuente: Base de datos CISDM. 
la diferencia entre medias de cada una de las variables independientes asociadas a los FFC activos, y los que quedaron inactivos después de la crisis. Para esto, como hipótesis nula se estableció que la diferencia entre las medias de las variables para los fondos activos e inactivos era igual a 0 , con la finalidad de determinar si existían diferencias estadísticamente significativas entre el grupo de FFC activos e inactivos.

Tabla 2.

P-valores resultantes de la prueba estadística de diferencia de medias entre variables asociadas a los FFC activos y los que quedaron inactivos después de la crisis

\begin{tabular}{|c|c|}
\hline Variable & P-Valor \\
\hline Autocorrelación de los rendimientos & 0,081 \\
\hline Curtosis & 0,107 \\
\hline Edad del fondo & 0,022 \\
\hline Frecuencia de redención & 0,456 \\
\hline Periodo para anuncio de redención & 0,398 \\
\hline El fondo usa financiamiento & 0,776 \\
\hline Porcentaje de incentivo & 0,044 \\
\hline Comisión por gerencia del fondo & 0,001 \\
\hline Inversión mínima & 0,205 \\
\hline Sesgo & 0,009 \\
\hline Auditado & 0,276 \\
\hline Rendimientos promedio antes de la crisis & 0,000 \\
\hline Desviación de los rendimientos & 0,024 \\
\hline Tamaño & 0,079 \\
\hline
\end{tabular}

Fuente: Base de datos CISDM.

Con base en estos resultados, puede verse que las diferencias entre las medias fueron estadísticamente significativas al $1 \%$ para las variables: comisión por gerencia del fondo, sesgo y rendimientos promedio antes de la crisis; al $5 \%$ para las variables edad del fondo, desviación de los rendimientos y porcentaje de incentivo; y al 10\% para las variables autocorrelación de los rendimientos y tamaño.

En la tabla 3 se presenta un análisis de las estadísticas descriptivas de los FFC, comparando el valor de las variables microeconómicas entre los fondos activos e inactivos. Esta tabla permite realizar comparaciones de los valores de cada una de las variables independiente entre los fondos activos e inactivos; sin embargo, tal comparación debe hacerse con cautela, puesto que no se está controlando por el efecto conjunto que las demás variables independientes pudieran tener sobre la variable dependiente, algo que se hará posteriormente cuando se realice la regresión del modelo Probit.

Se pueden apreciar ciertas diferencias entre la media, la desviación estándar y la mediana de algunas variables, como es el caso de los rendimientos promedio, los cuales, para los fondos activos, eran de $0,4 \%$ al mes, mientras que para los inactivos fue de $0,2 \%$ al mes. Además, para esta variable se observó un valor máximo de $0,36 \%$ al mes para los fondos activos, mientras que para los inactivos el valor máximo alcanzado fue de solo $0,18 \%$ al mes.

Al revisar cada una de las variables incluidas en el estudio, se tiene que para la autocorrelación de los rendimientos se obtuvo una media y una mediana mayor para los FFC activos respecto a los inactivos, mientras que la desviación estándar de los rendimientos fue menor para los fondos activos que para los inactivos, resultados que podrían explicar cómo aquellos FFC cuyos rendimientos eran menos volátiles tenían más probabilidades de sobrevivir a la crisis.

Respecto al coeficiente de curtosis, tanto la media como la desviación estándar y la mediana arrojaron valores inferiores para los fondos activos en comparación con los inactivos, lo cual indica que la distribución de los rendimientos para los fondos inactivos se ajustaba más a una curva leptocúrtica, es decir, se encontraban más concentrados alrededor de su media, lo cual es de esperar puesto que este grupo de fondos fue precisamente el que sobrevivió. Sin embargo, la mediana para esta variable arrojó un valor negativo tanto para los fondos activos como para los inactivos, lo cual permite sugerir que en la mayoría de los fondos sus rendimientos se ajustaban a curvas platicúrticas, es decir, con valores menos concentrados en la media.

Al observar la variable edad del fondo, se puede inferir, con base en los valores obtenidos para la media y la mediana - estos fueron mayores para los FFC activos-, que aquellos fondos de mayor edad tuvieron una mayor capacidad para 


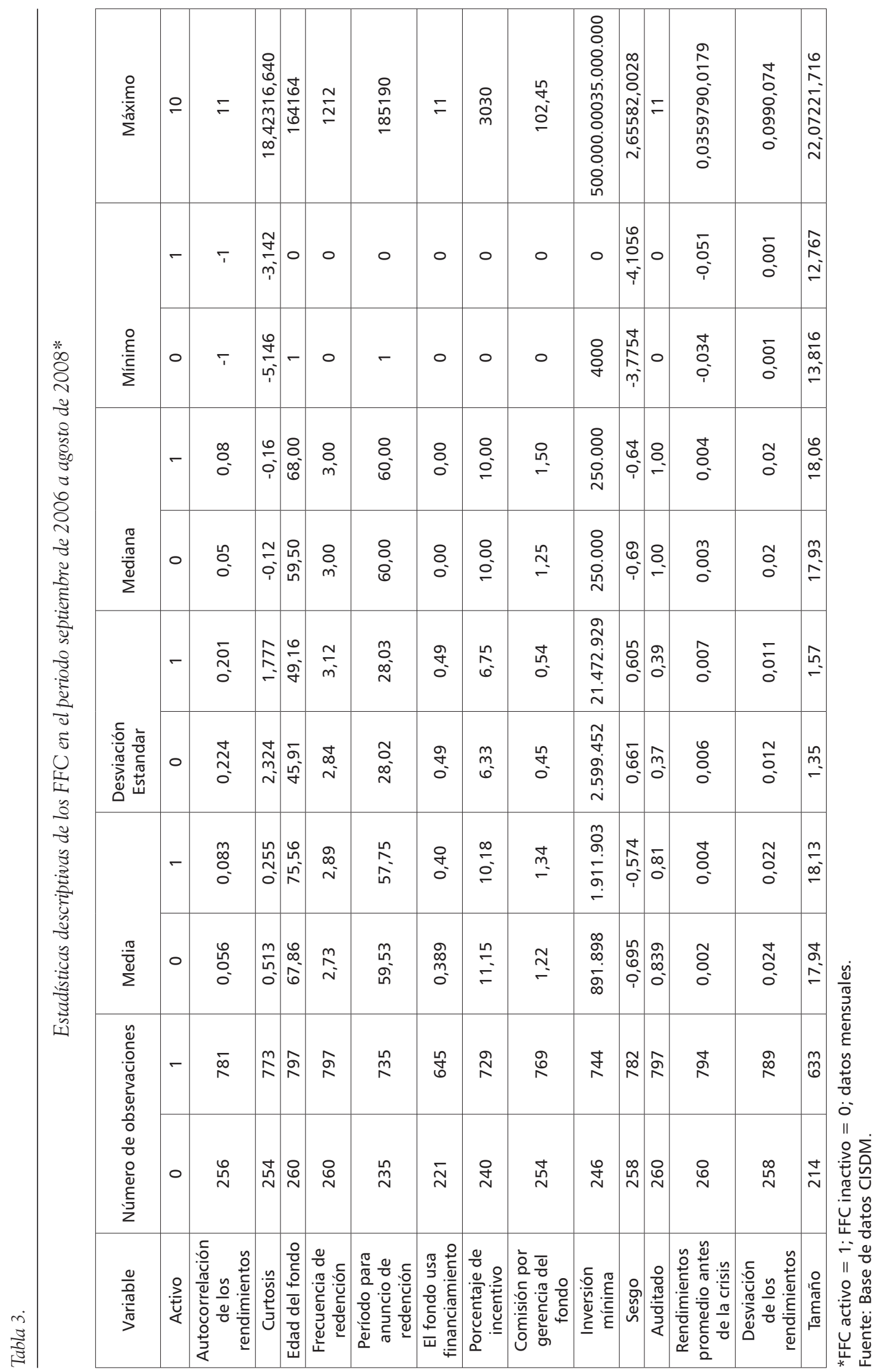


sobrevivir a la crisis y, por ende, encontrarse activos después de ella, lo cual es un resultado bastante lógico, debido a la experiencia acumulada por estos fondos.

Respecto a las variables frecuencia de redención y periodo para el anuncio de la redención, no se observaron diferencias marcadas entre los dos grupos de fondos. Inclusive la mediana de esta variable arrojó un valor igual tanto para los fondos activos como inactivos, a partir de lo cual puede concluirse que estos resultados no muestran evidencias suficientes (al menos al analizarlas sin tomar en cuenta el efecto conjunto del resto de las variables) para considerar la influencia de estas variables sobre el comportamiento de los FFC.

En relación con la variable financiamiento, tanto para la media como para la desviación estándar se obtuvieron valores muy similares; la mediana fue igual a 0 en ambos casos, lo cual sugiere que la mayoría de los fondos no se encontraba usando financiamiento el mes previo a la crisis. Ello no es de sorprender, puesto que los FFC hacen un uso menor del financiamiento en comparación con los FC.

Al analizar la variable porcentaje de incentivo, se puede apreciar que la media para los fondos inactivos fue ligeramente mayor; sin embargo, la mediana para ambos casos tuvo el mismo valor. Un porcentaje de incentivo más alto puede incentivar a algunos gerentes a tomar riesgos excesivos, poniendo en peligro la viabilidad del fondo. Al analizar la variable comisión por gerencia del fondo, se encontró una media y mediana mayor para el grupo de fondos activos, a partir de lo cual se puede inferir que aquellos fondos cuya gerencia era mejor $y$, por lo tanto, pudieron sobrevivir a la crisis cobraban un mayor porcentaje por su administración.

En relación con la variable inversión mínima, se observa una media muy superior para los fondos activos respecto a los inactivos. Ello muestra que este grupo de fondos era más selectivo en cuanto a los montos para el ingreso de nuevos inversionistas. Por su parte, al analizar la variable sesgo, los fondos activos reflejaron una media y mediana mayor que los inactivos, mientras que la desviación fue menor para el grupo de fondos activos.
En cuanto a la variable auditado, no se apreciaron mayores diferencias entre la media, desviación estándar y mediana, lo cual, contrariamente a lo que podría esperarse, no parece ser un factor diferenciador entre el grupo de fondos activos e inactivos. Esta variable ha sido determinante como predictor en el caso de los FC. Para los FFC tal vez sea menos importante debido a que estos vehículos invierten en unos fondos, los FC, que en su mayoría ya han sido auditados.

Al observar la variable desviación de los rendimientos, se puede apreciar que los fondos pertenecientes al grupo de inactivos tuvieron una media mayor, lo cual indica que aquellos fondos cuyos rendimientos eran más variables $y$, por lo tanto, más volátiles, fueron menos efectivos para mantenerse activos luego de la crisis.

Por último, al comparar los valores descriptivos para la variable tamaño, no se observaron diferencias marcadas entre la media y la mediana; inclusive los valores máximos y mínimos son muy similares. En este sentido, podría inferirse que esta variable no tuvo una influencia significativa en que el fondo estuviese activo o inactivo luego de la crisis.

\section{Modelo de regresión Probit}

A continuación, se llevó a cabo una regresión Probit con la intención de determinar qué variables independientes eran capaces de predecir si un FFC se encontraría activo o inactivo luego de la crisis. En este sentido, al observar la tabla 4, se puede apreciar que las siguientes variables fueron significativas: curtosis (P-valor de 0,028), porcentaje de incentivo (P-valor de 0,054$)$, comisión por gerencia del fondo (P-valor $<0,0001)$, rendimientos promedio antes de la crisis (P-valor $<0,0001$ ) y desviación estándar de los rendimientos (P-valor de 0,013). A continuación, se ofrece una interpretación de estos resultados.

Al analizar la variable curtosis, el coeficiente estimado en el modelo de regresión es de -0,091, lo cual indica que para valores inferiores a 0 , esta contribuye a que la variable dependiente se 
acerque al valor 1 (es decir, que el fondo permanecería activo). De esta forma, puede entenderse que aquellos fondos cuyos rendimientos previos a la crisis seguían una curva de distribución del tipo platicúrtica fueron más propensos a mantenerse activos luego de la crisis financiera.

La variable porcentaje de incentivo arrojó, en el modelo de regresión, un coeficiente negativo, lo cual sugiere que mientras mayor sea ese porcentaje, mayor será la probabilidad de que el FFC no permanezca activo luego de la crisis. Esto coincide con el análisis descriptivo realizado a los dos grupos de FFC, activos e inactivos, en el cual la media para esta variable era mayor para el grupo de fondos inactivos. Este resultado es lógico por la siguiente razón: un mayor porcentaje de incentivo significa que el gerente del FFC cobrará una mayor cantidad de dinero por cada dólar de ganancia obtenida. Así, es de esperar que mientras mayor sea este porcentaje, mayores sean los riesgos de que el gerente esté dispuesto a asumir, puesto que en caso de obtener ganancias para el fondo, percibirá una mayor remuneración.

Por otra parte, respecto a la variable comisión por gerencia del fondo, la cual se calcula como un porcentaje fijo de todos los activos gestionados, tiene un coeficiente positivo de 0,555 , lo cual indica que a mayores porcentajes de comisión por gerencia del fondo, mayores son las probabilidades de que el fondo se mantenga activo. Esto es consistente con los resultados del análisis descriptivo realizado con esta variable, en el cual su valor medio era estadística y significativamente mayor para el grupo de FFC activos. La comisión por gerencia del fondo es un porcentaje fijo, independiente del desempeño del fondo. $Y$ es de esperar que fondos con mayor reputación logren cobrar un porcentaje más alto, por los servicios que prestan a sus clientes y por su mejor desempeño percibido. Se puede esperar entonces que los fondos con mayor comisión por gerencia tengan una menor probabilidad de pasar a estar inactivos.

En relación con la variable de rendimiento promedio antes de la crisis, se obtuvo un P-valor casi igual a 0 , lo cual nos indica su incidencia significativa en el comportamiento de la variable dependiente. Asimismo, el coeficiente estimado por el modelo es positivo, lo cual muestra que a mayores rendimientos antes de la crisis, mayores son las probabilidades de que el FFC se encuentre activo luego de esta. Así, al comparar este resultado con los obtenidos, tanto en el análisis descriptivo como en la prueba de diferencia de medias, se observa cómo todos son consistentes, lo cual recalca la gran incidencia que tuvo esta variable sobre la condición de los FFC luego de la crisis. De manera similar, Xu, Liu y Loviscek (2011) concluyen en su estudio que mientras mejor sea el rendimiento en el periodo antes de la crisis, mayores serán las probabilidades de que el fondo se mantenga activo.

Para la variable desviación estándar de los rendimientos, el coeficiente estimado fue negativo; se entiende que a mayores valores de esta variable, mayores serán las probabilidades de que la variable dependiente tome el valor 0 . En otras palabras, aquellos FFC cuyos rendimientos fueron más volátiles, durante los 24 meses previos a la crisis, tuvieron una mayor propensión a quedar inactivos luego de esta, lo cual es de esperar si se considera que una mayor volatilidad de los rendimientos está asociada a una mayor probabilidad de tensión financiera para el fondo. Al igual que con la variable rendimientos promedio antes de la crisis, los resultados obtenidos del análisis descriptivo y de la prueba de diferencia de medias son consistentes con los obtenidos en la regresión.

Al analizar la variable edad, en principio era de esperarse que para FFC más antiguos las probabilidades de mantenerse activo fueran mayores. Sin embargo, no se encontró evidencia favorable a esta hipótesis al analizar los resultados del modelo de regresión Probit (la variable arrojó un coeficiente no significativo). Este resultado contrasta con el obtenido por Xu, Liu y Loviscek (2011) para los FC (recuérdese que aquí se están analizando FFC, y no los FC propiamente): desmostraron que esta variable sí tenía una influencia positiva sobre el comportamiento de los FFC durante la crisis. El surgimiento de un número creciente de FFC a lo largo de los años previos a la crisis y con niveles 
Tabla 4.

Resultados del modelo de regresión Probit

\begin{tabular}{|c|c|}
\hline \multicolumn{2}{|c|}{ Model Information } \\
\hline Data Set & TEG.FFC 08 \\
\hline Response Variable & Active \\
\hline Number of Response Levels & 2 \\
\hline Model & binary probit \\
\hline Optimization Technique & Fisher's scoring \\
\hline
\end{tabular}

\begin{tabular}{|c|c|}
\hline Number of Observations Read & 1057 \\
\hline Number of Observations Used & 586 \\
\hline
\end{tabular}

\begin{tabular}{|c|c|c|}
\hline \multicolumn{3}{|c|}{ Response Profile } \\
\hline Ordered Value & Active & Total Frequency \\
\hline 1 & 0 & 155 \\
\hline 2 & 1 & 431 \\
\hline
\end{tabular}

\begin{tabular}{|c|c|c|}
\hline \multicolumn{3}{|c|}{ Model Fit Statistics } \\
\hline Criterion & Intercept Only & $\begin{array}{c}\text { Intercept and } \\
\text { Covariates }\end{array}$ \\
\hline AIC & 679,084 & 655.768 \\
\hline SC & 683,457 & 721.368 \\
\hline-2 Log L & 677,084 & 625.768 \\
\hline
\end{tabular}

\begin{tabular}{|c|c|c|c|}
\hline \multicolumn{5}{|c|}{ Testing Global Null Hypothesis: BETA=0 } \\
\hline Test & Chi-Square & DF & Pr>ChiSq \\
\hline Likelihood Ratio & 51,316 & 14 & $<.0001$ \\
\hline Score & 48,4178 & 14 & $<.0001$ \\
\hline Wald & 44,6169 & 14 & $<.0001$ \\
\hline
\end{tabular}

\begin{tabular}{|c|c|c|c|c|c|}
\hline \multicolumn{7}{|c|}{ Analysis of Máximum Likelihood Estimates } \\
\hline Parameter & DF & Estimate & $\begin{array}{c}\text { Standard } \\
\text { Error }\end{array}$ & $\begin{array}{c}\text { Wald Chi- } \\
\text { Square }\end{array}$ & P-Value \\
\hline Intercept & 1 & 0,0766 & 0,8093 & 0,009 & 0,9246 \\
\hline Curtosis & 1 & 0,2699 & 0,3379 & 0,6383 & 0,4243 \\
\hline Eutocorrelación de los rendimientos & 1 & $-0,091$ & 0,0414 & 4,8251 & 0,028 \\
\hline Frecuencia de redención & 1 & $6,65 \mathrm{E}-06$ & 0,00131 & 0 & 0,996 \\
\hline Periodo para anuncio de redención & 1 & 0,0145 & 0,0192 & 0,5669 & 0,4515 \\
\hline El fondo usa financiamiento & 1 & $-0,00191$ & 0,00234 & 0,6694 & 0,4133 \\
\hline Porcentaje de incentivo & 1 & 0,0147 & 0,1223 & 0,0145 & 0,9041 \\
\hline Comisión por gerencia del fondo & 1 & $-0,0173$ & 0,00892 & 3,7625 & 0,0524 \\
\hline Inversión mínima & 1 & $7,03 \mathrm{E}-09$ & $1,09 \mathrm{E}-08$ & 0,4197 & 0,5171 \\
\hline Sesgo & 1 & $-0,0932$ & 0,1295 & 0,518 & 0,4717 \\
\hline Auditado & 1 & 0,1036 & 0,1724 & 0,361 & 0,548 \\
\hline Rendimientos promedio antes de la crisis & 1 & 55,6288 & 13,8577 & 16,1145 & $<.0001$ \\
\hline Desviación de los rendimientos & 1 & $-13,1938$ & 5,3133 & 6,166 & 0,013 \\
\hline Tamaño & 1 & 0,00108 & 0,0433 & 0,0006 & 0,98 \\
\hline
\end{tabular}

\begin{tabular}{|c|c|c|r|}
\hline \multicolumn{4}{|c|}{ Association of Predicted Probabilities and Observed Responses } \\
\hline Percent Concordant & 68 & Somers'D & 0,366 \\
\hline Percent Discordant & 31,5 & Gamma & 0,367 \\
\hline Percent Tied & 0,5 & Tau-a & 0,142 \\
\hline Pairs & 66805 & c & 0,683 \\
\hline
\end{tabular}

Fuente: 
cada vez mayores de diversificación (al diversificar de manera creciente en varias estrategias de FC, y no en una sola estrategia) pudiera explicar que los nuevos FFC tuviesen mayores posibilidades de sobrevivir a la crisis.

De igual forma ocurrió con la variable auditado. Xu, Liu y Loviscek (2011) consiguieron que aquellos FC que eran auditados sobre una base regular y declaraban tener una compañía auditora durante el periodo previo a la crisis tenían mayores probabilidades de mantenerse activos. Sin embargo, en el modelo Probit propuesto no se consiguió evidencia de que dicha variable fuera significativa sobre el comportamiento de la variable dependiente, en el caso de los FFC. Este resultado se puede explicar por el hecho de que los FFC, al invertir en FC individuales que en su mayoría ya se encuentran auditados, cuentan con una especie de protección previa con respecto a las auditorías, puesto que la mayoría de los FFC también están a su vez auditados.

Respecto a si el fondo usa financiamiento, a pesar de que se esperaba que los FFC apalancados fueran más susceptibles a condiciones de mercado desfavorables, no mostró ser una variable significativa en el modelo de regresión Probit, lo cual coincide con la conclusión de Xu, Liu y Loviscek (2011) para los FC.

Por último, el resto de las variables independientes (autocorrelación de los rendimientos, frecuencia de redención, periodo para redención, inversión mínima, sesgo y tamaño) no fueron estadísticamente significativas en el modelo de regresión Probit.

\section{CONCLUSIONES}

La crisis financiera global de 2008-2009 se considera como la peor desde la Gran Depresión de la década de los treinta, al asestar un duro golpe a las economías de los países desarrollados y al tener también un efecto negativo, aunque más breve, sobre los países en desarrollo. La crisis se hizo evidente en septiembre de 2008, con la quiebra de Lehman Brothers y el desplome de las bolsas de valores que se inició ese mes y que continuaría hasta marzo de 2009, cuando se alcanzaron los niveles más bajos en los principales índices bursátiles de Estado Unidos. La caída de las bolsas había estado precedida por la disminución de los precios de los inmuebles que ya se había iniciado en Estados Unidos, la cual afectó severamente a los títulos que habían sido emitidos en niveles récord basados en hipotecas.

La quiebra de Lehman Brothers en septiembre de 2008 fue desastrosa para muchos FC, puesto que este banco de inversión era el principal corredor primario de muchos de estos fondos. A ello se añadió el efecto negativo que la caída abrupta de las bolsas de valores ocasionó en las valoraciones de las carteras de los FC. Ello condujo en no pocos casos al cierre del FC respectivo, creando una gran disrupción en los FFC, los cuales invierten en FC.

La principal innovación de este trabajo consistió en la implementación de un modelo Probit para intentar explicar la probabilidad de que los FFC (en lugar de los FC, ya estudiados por Xu, Liu y Loviscek [2011]) hubieran permanecido activos después de la crisis de 2008-2009. Adicionalmente, en el modelo Probit propuesto se consideraron algunas variables que no habían sido contempladas en el trabajo de los autores mencionados. Esas nuevas variables fueron: autocorrelación de los rendimientos, curtosis y sesgo de la distribución, frecuencia de redención, periodo para anuncio de redención, porcentaje de incentivo, comisión por gerencia del fondo e inversión mínima en el fondo.

La estrategia seguida por los FFC resultó ser un factor determinante para su desempeño durante la crisis financiera de 2008-2009, puesto que los FFC que se mantuvieron activos luego de esta aplicaron en su mayoría una multiestrategia, lo cual se vio reflejado en que sus rendimientos se distribuyeron en una curva menos concentrada alrededor de su media, sugiriendo así que eran más flexibles, para adaptarse mes a mes, a los cambios del mercado.

El rendimiento promedio registrado por los FFC durante el periodo previo a la crisis de 20082009 fue esencial para poder explicar que los 
fondos se mantuvieran activos luego de ella. El que los FFC activos obtuvieran mejores rendimientos previo a la crisis, les permitió hacer frente a la crisis con una mayor fortaleza financiera y les ayudó a sobrevivir a esta. Además, aquellos FFC con menor volatilidad en sus rendimientos, representada por la variabilidad de los mismos durante los 24 meses previos a la crisis, mostraron una mayor capacidad para poder mantenerse activos.

Los FFC con comisiones por gerencia (el cual es un porcentaje fijo calculado sobre el total de activos gestionados) más altas mostraron tener una mayor probabilidad de sobrevivir a la crisis financiera, gracias a la capacidad que pudieron haber tenido para captar mejores gerentes que otros FFC. Sin embargo, el porcentaje de incentivo para el gerente del FFC mostró tener una influencia negativa sobre su desempeño, lo cual puede explicarse como un factor que derivó en decisiones y estrategias más arriesgadas y de mayor exposición por parte del gerente, con el objetivo de superar su benchmark, aunque poniendo en una situación de mayor riesgo al FFC.

Por último, las siguientes variables microeconómicas no mostraron evidencia de influir significativamente sobre el desempeño de los FFC durante la crisis financiera global: edad del fondo, frecuencia de redención, periodo de redención, financiamiento, inversión mínima, auditado y el tamaño.

El presente estudio pudiera ser expandido en varias direcciones. En primer lugar, se pudiera volver a examinar el efecto de la crisis de 20082009 sobre los FFC, pero empleando otras ventanas de tiempo. En segundo lugar, sería de utilidad intentar aplicar el modelo Probit aquí propuesto a otros eventos de crisis, aunque considerando que ninguno de ellos ha tenido la severidad de la crisis de 2008-2009. La crisis de agosto de 1998, cuando el FC Long-Term Capital Management se fue a la bancarrota, en principio representaba un evento de crisis relevante para ser analizado, aunque se pudo comprobar que había muy pocos FFC en ese momento como para poder realizar un estudio cuyas conclusiones llegasen a ser sólidas. En tercer lugar, se pudiera aplicar el modelo Probit aquí propuesto a los FC (en vez de a los FFC, como ya se hizo aquí), considerando que en este trabajo se propusieron nuevas variables explicativas de la probabilidad de sobrevivencia, las cuales no han sido aplicadas en la literatura existente a los FC, hasta donde se ha podido comprobar.

\section{REFERENCIAS}

1. Acharya, V. y M. Richardson (2009). Causes of the financial crisis. Critical Review, 21(2-3), 195-210.

2. Acharya, V., Philippon, T., Richardson, M. y Roubini, N. (2009). The financial crisis of 2007-2009: Causes and remedies. Financial Markets, Institutions $\mathcal{E}$ Instruments, 18(2), 89-137.

3. Amonlirdman, K., Getmansky, M., Kurma, R. y Lo, A. W. (2004). The dynamics of global financial crises. Massachussets: MIT Sloan School of Management.

4. Billio, M, Getmansky, M. y Pelizzon, L. (2009). Crises and hedge fund risk. Amherst: Universidad de Massachusetts.

5. Bollen, N. P., y. Do Pool, V. K, (2009). Hedge fund managers misreport returns? Evidence from the pooled distribution. The Journal of Finance, 64(5), 2257-2288.

6. Bollen, N. P. y Whaley, R. E. (2009). Hedge fund risk dynamics: implications for performance appraisal. Journal of Finance, 64, 987-1037.

7. Covitz, D., Liang, N. y Suárez, G. (2009). The anatomy of a financial crisis: The evolution of panic-driven runs in the asset-backed commercial paper market. Working Paper, Board of Governors of the Federal Reserve. 
8. Donolue, M., Garay, U., Jaffrain, E., L'habitant, F., Piere-Yves Mathonet, T., Spurgin, y R. Stevenson (2009). CAIA Level II Advanced Core Topics in Alternative Investments. John Wiley \& Sons.

9. Fama, E, y K. French (1992). The cross-section of expected stock returns. The Journal of Finance, 47(2), 427-465.

10. Fama, E, y K. French (1993). Common risk factors in the returns on stocks and bonds. Journal of Financial Economics, 33, 3-56.

11. Fung, W. y Hsieh, y D. A. (2002). Asset-based Style Factors for Hedge Funds. Financial Analyst Journal, 58(5), 16-27.

12. Fung, W. y Hsieh, D. A. (2004). Hedge fund benchmarks: a risk based approach. Financial Analyst Journal, $60,65-80$.

13. Fung, W., Hsieh, D. A., Ramadorai, T. y Naik, N. (2008). Hedge funds: performance, risk, and capital formation. The Journal of Finance, 63(4), 1777-1803.

14. Garay, U., Molina, G., Rodríguez, A. y Ter Horst, E. (2016). Bayesian Non-Parametric Estimation of Hedge Fund Performance. Econometrics, 4, 13.

15. Garay, U. y Ter Horst, E. (2009). Real estate and private equity: A review of the diversification benefits and some recent developments. Journal of Alternative Investments, 11 (4), 90-101.

16. Getmansky, M., Lee, P. y Lo, A. (2015). Hedge funds: A dynamic industry in transition. Recuperado de http://ssrn.com/abstract $=2637007$

17. Ivashina, V. y Scharfstein, D. (2010). Bank lending during the financial crisis of 2008. Journal of Financial Economics, 97, 319-338.

18. Levitin, A. y Wachter, S. (2012). Explaining the housing bubble. Georgetown Law Journal, 100(4), $1177-1258$.

19. Mian, A. y Sufi, A. (2011). House Prices, Home Equity-Based Borrowing, and the US Household Leverage Crisis American Economic Review, 101 (5), 2132-2156.

20. Sharpe, W. (1992). Asset allocation: management style and performance measurement. Journal of Portfolio Management, 18, 7-19.

21. Stulz, R. M. (2007). Hedge funds: past, present, and future. Journal of Economics Perspectives, 21 (2), 175-194.

22. Xu, E., Liu, J. y Loviscek, A. (2011). An examination of hedge fund survivorship bias and attrition before and during the global financial crisis. Journal of Alternative Investments, 13(4), 40-52. 